\title{
Aligning academic medicine within the healthcare system: the APS-SPR virtual chat series
}

\author{
Thomas Shanley ${ }^{1,2}$, Allison Brashear ${ }^{3,4}$ and S. Claiborne Johnston ${ }^{5}$ \\ (c) The Author(s), under exclusive licence to the International Pediatric Research Foundation, Inc 2022
}

Pediatric Research (2023) 93:503-510; https://doi.org/10.1038/s41390-022-01963-7

Steve Abman (SA) Welcome to the 10th and final session of our APS-SPR Joint Virtual Chat Series on "Challenges in Academic Medicine". This series was designed to provide an ongoing forum to discuss diverse and critical issues impacting academic pediatrics. The purpose of this series was to create a structured, yet informal, approach that would encourage direct dialog in a conversational format, involving leading academic pediatric experts. Diverse members of the pediatric community across multiple disciplines and across the full range of the "pipeline" or academic lifespan are part of the "virtual conversation".

Today's session is entitled, "Aligning Academic Medicine within the Academic Healthcare System". Successfully achieving our goals of our academic missions, especially in regard to education and training, research, advocacy, and clinical care, are highly dependent on the relationships between our medical schools, affiliated hospitals, and business strategy departments. Balancing fiscal challenges with these academic pursuits requires many talents, as well as strong and visionary leadership. Understanding this interface is essential for our successes in academic medicine. We are excited to have three outstanding leaders with expertise and track records regarding these complex issues.

Dr. Allison Brashear is Professor of Neurology and Dean of the School of Medicine at the University of California Davis. Dr. Brashear completed medical school and neurology residency at Indiana University, where she joined the faculty and became an internationally-renowned investigator in movement disorders. She served as Chair of Neurology at Wake Forest for 14 years, after which she obtained her MBA at Duke University, with a focus on health sector management along with special expertise in health policy, and hospital-clinical integration. Dr. Brashear serves on many boards, including the McKnight Brain Research Foundation, the American Board of Psychiatry and Neurology, and the California Institute of Regenerative Medicine (CIRM).

Dr. Clay Johnston is Dean of the Dell Medical School of Medicine and holds the Frank and Charmaine Denius Distinguished Dean's Chair at the University of Texas in Austin. Dr. Johnston is also a Neurologist. He completed medical school at Harvard, his Neurology Residency and a stroke fellowship at UCSF, followed by additional training at Berkeley, where he obtained his Ph.D. in epidemiology. In addition to serving as Director of the Clinical and Translational Science Institute at UCSF, Dr. Johnston has had a remarkable academic career, with an impressive and extensive track record of publications and grants that are largely focused on stroke. He has received many awards throughout his career and serves in many national leadership positions.

Finally, Dr. Tom Shanley is a Professor of Pediatrics at Northwestern University and is President and CEO of Ann \& Robert H. Lurie Children's Hospital. Dr. Shanley trained in Pediatric Critical Care Medicine as part of the Pediatric Scientist Development Program at the University of Michigan before starting his faculty career at Cincinnati Children's Hospital Medical Center. He was recruited back to the University of Michigan to serve as Director of Critical Care and later became Director of the CTSAsupported Translational Research Institute. He then served as Chair of Pediatrics and Chief Research Officer at Lurie Children's Hospital. In 2019, he became President and Chief Executive Officer of Ann \& Robert H. Lurie Children's Hospital of Chicago.

Stephanie Davis (SD) We will start today's "virtual chat" with Dr. Shanley.

Thomas Shanley (TS) As it relates to the alignment of academic medicine to healthcare systems, I want to make sure that we are using common language as we go through the discussion. When we say "academic medicine" | am generally including those activities that extend beyond revenue-generating clinical activities. We most often think of the research mission, the education and training mission, and the advocacy mission.

Each of these domains has some identifiable sources of revenue to support them. Obviously, external grants can be used to support the expenses of a research program but often they are not sufficient to support the entire program in terms of personnel, equipment, et cetera. In this case, the medical center may determine whether they want to sustain a program by subsidizing the funding or reduce or even stop the program.

Similarly, we know our training programs are dependent on GME funding for those that are part of integrated systems, or Children's Hospital GME funding for independent Children's Hospitals. GME funding provides some support for our residents and fellows. However, often, this funding doesn't cover the entire expense for the cohort of trainees at a particular institution.

In regard to advocacy, there is no external support for this critical mission, which occurs at both the state and federal level for many of our institutions. This includes advocating for policies that are critical to the revenue that supports our academic missions, like NIH funding, CHGME, or GME funding. To not participate in this type of advocacy can be costly.

\footnotetext{
'Department of Administration, Ann \& Robert H. Lurie Children's Hospital of Chicago, Chicago, IL, USA. ${ }^{2}$ Department of Pediatrics, Northwestern University Feinberg School of Medicine, Chicago, IL, USA. ${ }^{3}$ School of Medicine, University of California, Davis, CA, USA. ${ }^{4}$ Neurology, University of California, Davis, CA, USA. ${ }^{5}$ Dell Medical School, University of Texas at Austin, Austin, TX, USA. ${ }^{凶}$ email: TShanley@luriechildrens.org
} 
So, how do we align these activities within a "health care system"? It is important to define what we mean when we discuss a "health care system". There are various models that determine the important factors of how funds flow, and notably how clinical revenue streams flow, and which groups may have access to these funds. In regard to academic alignment, there's generally two structures that we identify. The first structure is one in which a children's hospital or a pediatric department is part of a larger university-based medical center. For example, C.S. Mott Children's Hospital is integrated within the broader University of Michigan Healthcare System. The second structure is the model of an independent freestanding self-governing children's hospital, for example, Lurie Children's Hospital. At Lurie Children's Hospital, we have an academic affiliation with Northwestern's Feinberg School of Medicine where faculty have appointments, but the rest of the operations are independent.

I recognize some of you may be at an institution that does not completely fit these two models. We know there are some children's hospitals that are a part of a larger non-academic health care system. For this discussion, these two models are the most common ones when we think about how to align academic medicine within healthcare systems.

To understand how various revenue streams are allocated, we need to review the two revenue streams generated. One revenue stream is from the physicians and other providers (e.g., APNs); these clinical services are reimbursed as professional service fees. The second revenue stream is the facility or hospital fees that the system receives due to providing services within their physical facilities. Prioritizing allocation of these financial resources to academic missions within an institution really depends upon the strategies that are defined and designed by each institution.

We will probably share personal perspectives during this discussion; therefore, I wanted to introduce what I typically refer to as the "Shanley bias". This bias is that I have always prioritized optimizing clinical outcomes. This priority focuses on being a leader at assuring the best clinical outcomes for children. If you commit to this priority, I have identified two necessary components.

The first component is ensuring that you are implementing the best evidence-based management approach associated with the leading outcome. Over the last couple of decades, we have focused on data transparency, consortium-level outcomes, and public reporting, and this has led to an opportunity to understand which peer institutions are performing at the highest level in terms of outcome measures. This requires significant investments in quality and safety programs, including outcome and process measurement tools, improvement tools, and personnel that are needed to lead those efforts. Each time you highlight an investment for alignment, I think you have to consider the return on that investment and how you assess that value.

When I think about our quality and safety measures, we focus on quantitative outcome measures that demonstrate improved care over time. This leads to money being saved through the prevention of complications. For example, consider a central lineassociated bloodstream infection. Recognize both the clinical value (clear reductions in length of stay and morbidity/mortality), as well as the costs you'll avoid for each bloodstream infection prevented. These clinical outcomes can provide your institution with market differentiation when families are shopping for where their child can receive the best care. In addition, there certainly is an impact when you optimize these clinical outcomes and reduce medical malpractice claims cost and malpractice premiums, especially when you're insuring yourself. You should be able to describe the outcome measures of impact in any quality and safety program.

The second component of "optimizing outcomes" is striving towards what I describe as a theoretical best. As a former pediatric ICU physician who treated children with sepsis, my theoretical best outcome is $100 \%$, that is, every child survives their episode of sepsis. To move from one's current best outcome to what we describe as this theoretical, optimal best, leveraging discovery and innovation is important. This may be in diagnostics, an improved mechanistic understanding, or novel therapeutics. It is that investment in the research and discovery enterprise that can fundamentally change how we understand and subsequently manage any particular disease. This change in understanding the disease and in management advances the outcome towards an optimal best.

As we think about these investments, you are also thinking about the return on investment. This return on investment may be measured through innovation, research funding success, and frankly, the clinical impact of those discoveries. At Lurie Children's, we now have a platform that measures the impact of our research along five domains. The message is that measuring what you receive from the investments in the research enterprise can be quantified. This impact can be qualified as well because of the stories of impact, the stories of lives that are changed.

The question becomes, how might this approach be applied to a particular disease or patient population? That really depends on what you prioritize within your healthcare system. More often than not, this really depends on three factors. The first factor is your mission. This defines your focus in terms of a particular patient cohort. The second factor is the market. You need to ensure that there is a patient volume for those services you are delivering. The third factor or the hard one, is the margin. What is the margin that you make when caring for the cohort? We know where we generate positive margins from the revenue stream generated by the clinical care minus the expenses to provide that clinical care. This is what really creates revenue to drive the rest of the investments across the medical system. These factors help to define the decisions that align academic medicine within the healthcare system: mission, market, and margin.

(SD) How does the healthcare system or hospital hear about opportunities to invest in the academic program?

(TS) It is a classic dichotomy question: Is this a top-down decision-making process, or is this an organic, bubble-up process? If you are considering the latter, how do you facilitate and encourage this type of process? I would say that most institutions conduct strategic planning. Incorporating frontline providers and frontline scientists into strategic planning provides an opportunity to engage them in a bubble-up process. That's how we structure this type of work in order to ensure that we have a broad-based engagement of voices and perspectives to share what they identify as the greatest opportunities.

This was the process we facilitated for strategic planning. In addition, I didn't feel at that time that we were positioned to build programs from scratch. Therefore, we believed that the necessary starting point for strategic planning was to bring the perspective of individuals who were connected to existing research programs together along with other partnership and scientific opportunities across the campus. In general, I think facilitating and creating opportunities for faculty input involves a number of e-mail threads and communication approaches that foster bidirectional communication. I think it's critical to solicit input from the frontline.

(SD) Having the right stakeholders at the table is important. We are now going to ask Dr. Brashear to speak.

Allison Brashear (AB) Thank you, Tom. I'm going to tell a story, which I think is really a great story about aligning academic medicine with health care, and unfortunately, it has to do with the recent pandemic. As you may remember, a little over a year ago, UC Davis had the first community-acquired case of COVID-19 in the country. I can remember, lines of TV station trucks on February 26th, 2020. On March 2nd, we had an "all hands-on deck" meeting with researchers from our primate center, pathologists, virologists, clinicians, the IRB, and those who do contracting. Everybody represented some portion of the clinical and research enterprise. 
In one hour, we decided that we were going to bring clinical trials directly to these patients and create our own COVID test. Please keep in mind that this was March 2nd, 2020, weeks before the mandatory statewide shutdown and the first wave of cases.

Within two weeks, we initiated two trials. In each trial, within five days of receiving the protocol from the company, we had IRB approval, contracting was complete and we were able to infuse the patient in our hospital. One patient received a Remdesivir infusion at 3 a.m. after receiving IRB approval. That was really the beginning of a hand-in-glove collaboration between the School of Medicine and the hospital. We developed a COVID test within two weeks of our March 2nd huddle. We then were able to test patients and offer them these life-saving clinical trials. I remember walking into our command center in late March 2020 and discussing how we were delivering drugs to patients that wouldn't have had these drugs without our research trials. It was just a game-changer in terms of the nurses and staff who were caring for these patients, to realize that this was an opportunity that we had because we are a research institution and that we could offer these therapies through clinical trials because we are part of an integrated healthcare system.

So, fast forward to our main campus, UC Davis in Sacramento, where a high-throughput test was developed based on knowledge within our School of Agriculture. We have a large undergraduate campus, about 35,000 students, 30 miles away from our medical school, hospital, and UC Davis Betty Irene Moore School of Nursing where I am sitting in Sacramento. In August of 2020, our new Provost, Dr. Mary Croughan, supported the development of a testing modality that was by Dr. Michelmore, a School of Medicine faculty member, using technology used in the UC Davis College of Agriculture. This was developed in about six weeks. The main campus at UC Davis was also able to promote high-throughput asymptomatic testing. Asymptomatic testing led to a widespread behavior change in the entire town of Davis. We brought that testing to our campus and then used the same scheduling technology to implement our vaccine delivery system here in the healthcare system. The entire package, testing, and vaccine scheduling technology were possible because of the partnership between the School of Medicine and our main campus. The story of testing the entire town of Davis was highlighted on January 30th, 2021 on the front page of the New York Times.

Our testing and clinical trial capability also ensured that we were able to enroll a diverse population in the Pfizer COVID vaccine trial. UC Davis School of Medicine partnered with our community to enroll 225 patients, representing 36\% Latinx, 15\% Asian, 10\% Black, and 6\% Native American, one of the most diverse populations to be enrolled in that trial in the country.

As I review these experiences from last year, experiences that really brought everybody together, I think about alignment with three main goals in mind: (1) health equity, (2) leveraging research as core to the mission of a School of Medicine, (As Tom said, the value proposition for why a patient comes to an academic medical center.) and (3) the development of multi-disciplinary programs that really focus on healthcare outcomes. By focusing on these three key principles, you can pivot the entire healthcare system to focus on how to keep people well.

At UC Davis, we are known for the diversity of our students. Our student population is about $30 \%$ Latinx and $12 \%$ African American. Half of our medical students go into primary care and $70 \%$ of our students are from socioeconomically disadvantaged backgrounds. We have developed a variety of pathway programs to welcome individuals from diverse backgrounds into medical school. The goal is to build a diverse physician workforce for Northern California. We really believe that's one of the ways we can change healthcare.

By training a diverse workforce, many of these students return to areas in Northern California that don't have physicians, and certainly don't have specialists. We try to make sure that the students are really engaged in the community. They have studentrun clinics, and many of them stay in California. We believe this is one of the ways that we can really improve health. We know that if you have the same cultural background as your physician, you are more likely to seek advice from the doctor and more likely to follow their advice. In academic medicine, I think this is how we can really align the school's academic missions, such as education, research, and clinical care, with the community.

I would also like to highlight the importance of integrating research and education directly to the bedside. So, in the story I just told, what I didn't mention was that we had 7 a.m. calls every day from this office, for months, and now, they are down to once a week. We have been conducting these calls for over 16 months. In real-time, we broke down barriers and facilitated enrolling patients into trials. One example was the day where at 7 a.m., we had no space for the vaccine clinical trial patients and by the end of the call at 7:30 a.m., we had identified the necessary space. This problem was rapidly solved because people began to see the value proposition of having research directly available to our patients. Now, we are trying to really expand so that every patient who enters our door is considered a possible enrollee in a research trial.

I'm pleased to say that we have a $\mathrm{NCl}$-designated comprehensive cancer center. That is so important for patients who can access clinical trials that are not available elsewhere. For academic medicine, we need to tell the story about why the patient with a single breast mass should come to a comprehensive cancer center, instead of only visiting the center when they have recurrent disease. They receive state-of-the-art care and access to research from the beginning.

I never thought there would be a time when most of the country would know who Tony Fauci is, what the $\mathrm{NIH}$ is, and have an understanding about research studies and placebo-controlled trials. In the story I told you about the vaccine trial, what I did not say is that there were 4000 people who signed up to be one of the 225. I believe we changed the conversation about the importance of research. We changed the conversation, certainly at the hospital, with the staff, nurses, and administrators. I think nationally UC Davis School of Medicine changed the conversation because the School of Medicine and the Academic Healthcare System really aligned to make a difference.

Finally, I would like to highlight the importance of focusing on programs that improve health. What are the outcome measures for which you make investments? At UC Davis, we are building an aging program to keep people healthy. This is a clinic designed for patients involved in this program. Patients in the aging program are immediately drawn to the research that we have for memory disorders. We've been able to partner with the hospital to design an age-friendly designation for our emergency room, and soon an age-friendly designation for our hospital. We are working on similar initiatives for psychiatric disease. You must get everybody on the same page, but you can make a big difference when you focus on building programs focused on a disease state. The result is that you keep people healthier, out of the hospital and hopefully decrease cost and improve outcomes. If you prevent a patient with behavioral health issues from going to the emergency room and from being admitted, then you are helping everyone: the patient, their family, and the healthcare systems. Similarly, building focused programs for those most at risk, such as those patients who make frequent trips to the ED or those with repeated hospital admissions due to the lack of a safety net, improves health and decreases costs.

Finally, I am really pleased to highlight telemedicine initiatives. We were doing 10 telemedicine visits a day before COVID, and we went increased to almost 950 at the peak of the pandemic. Overall, about $50 \%$ of the visits are telemedicine. It's really been a gamechanger for how we can improve access for so many patients. 
The skill to do this initiative came from our researchers in our school of medicine; they were really knowledgeable about how to implement telemedicine and they brought that knowledge to the healthcare system.

The takeaway is that we have shown that academic healthcare systems, schools of medicine, and hospitals, can work together to improve the health of the entire community. That's the bright spot amidst all the tragic loss in 2020. As we emerge from the pandemic, we want to make sure that the leadership continues to prioritize science first and bring it to our patients. I hope that we are all now seeing how valuable these partnerships are, and how we can really make a big difference in people's lives, improve health and certainly make these types of partnerships a role model for the future.

(SD) How do you develop programs that improve health in both an efficient and cost-effective manner?

(AB) Our UC Davis aging initiative is a great example. More resources are required to stand up a clinic and provide multidisciplinary care. However, this clinic keeps people from coming to the emergency room. It prevents them from getting admitted. The clinic sets up patients to be in a healthy state, and if they then need care from an orthopedic surgeon or a neurosurgeon, they are aligned with our healthcare system. I personally think sometimes we need to invest upfront in keeping people healthy. Our hospitals are already overburdened. By investing in health, keeping people from being admitted to the hospital, and decreasing emergency room visits, we create hospital and clinic capacity to care for others who urgently need the care. To do this, we need to invest in the patients who are most at risk. That is how we really move from volume to value and do so in a focused way to create the most return on the investment for those most at risk.

I had a patient in North Carolina, a neurology patient, who had a wonderful family, but experienced multiple re-admissions due to Dilantin toxicity. We put her in our post-discharge program. As part of this program, she had intensive interventions, assuring that she had all the right resources. She never was admitted there again. She and her family benefitted from her staying out of the hospital. Because she wasn't admitted, this saved costs and created capacity in the healthcare system.

(SD) We are now going to ask Dr. Johnston to speak.

Clay Johnston (CJ) I really enjoyed Allison and Tom's comments. I agree completely with Allison about COVID being an opportunity for us to rethink how we align these missions and push them forward. I do think we should celebrate how well we have done nationally, with the progress in clinical care and in vaccines.

I am going to dial back the clock before COVID to highlight a couple of stories. These stories occurred in different settings, where initiatives have not worked so well. I would like to highlight insights that I gained leading to a change in how I approach work. We are a brand-new medical school. Also, we do not own our hospital and that's been an interesting adventure.

Before this position, I was at UCSF, and led the Clinical Translational Science Institute, the CTSI. I had a great job. At UCSF, the hospital is led by a Hospital CEO. There is also the Dean and they both report to the Chancellor. Given this, there is some alignment. There are many systems that are structured in a similar way to UCSF. As the lead of the CTSI, I was in between them, battling with the hospital about resources and space and other issues.

I had several meetings with the CEO, a great guy, Mark Laret. One of the issues that we discussed was how the hospital could better support clinical research. How do we engage every patient as a potential research subject? How do we make the data systems work more efficiently? How do we make patient recruitment a key piece of the practice? It was a whole series of conversations. A couple of important insights emerged from these conversations.

One insight was that Mark told me that he did not understand research and did not understand the value. All his incentives were based on the hospital margin. It was his job to turn the hospital margins over to the Dean. He would hold onto some margin to invest in the hospital, but the rest goes to the Dean. The Dean then decides how to invest in research and education with the proceeds from the clinical enterprise. So, his take is, unless you are presenting initiatives that increase the hospital margin, he has to think twice about the value. Basically, he was saying to speak to the Dean about these initiatives. This is a really important perspective. He was a wonderful guy, but he had clearly defined goals, which were distinct from advancing research. However, there was common ground.

One area of common ground was that he recognized that the hospital's reputation was almost entirely built on the research enterprise. Of course, the clinical care is great. However, the rankings and the reputation in the community is largely built on clinical research prowess. He recognized that research was important, and he was glad that work was being done.

He also had concerns about how the research was being conducted. This is nicely encapsulated in another conversation that taught me about an operational focus. I said to him that we needed more resources to conduct research, mainly IT resources. He responded by asking for something from me. He said, "You have 16 acute care beds and our hospital is always full. Each bed is probably worth, \$2 to \$3 million a year. You don't even keep these beds full, right?" He was referring to our Clinical Research Center. We tried to keep the beds full; however, there were empty beds some of the time. His question and ask were, "Do you really need all those beds? I will pay you to move out." It was an interesting conversation, highlighting how disconnected we were. I inherited the clinical research center, which had been there for decades and had been underutilized. Is there an opportunity for synergy? There's also the political battle of actually ceding control of the space.

So, at UCSF, even though everybody reports to the same ultimate boss, the incentives are not the same. You have to really get underneath what someone's telling you, to understand what incentivizes them. Try to find common ground to move initiatives forward. That was my lesson.

There was no Dean before me at Dell Medical School of Medicine; there also had been no medical school. This institution had been a community hospital system. There were two systems that competed. One was the Catholic system that took over the County Hospital years ago, so this was the Safety Net System. There was also a HCA system. You can guess which system was our partner. They were wonderful in their alignment with the goal of taking care of the population, but they didn't know about research. Their focus had been on physician parking, good food for the physicians, and bringing cases to the hospital.

What were we about? Why did they even want to work with us? Branding? They knew that the UT brand was a powerful one in Texas, and that the longhorn logo is the most recognized internationally of any school symbol. Even if they can't quite make it through the basketball tournament, they're still well recognized and so that's what interested the hospital system. The system would contribute financially to the residents, and that was about all, to capture us and keep us.

Then, we had a breakthrough. First, there was a change in system leadership: someone who understood the importance of academic medicine. Second, we had a physician, Chuck Fraser, an internationally prominent pediatric cardiovascular surgeon, who really wanted to join the faculty. He was only going to come if he worked for the medical school and if everyone that worked with him worked for the medical school. Through this arrangement, he could be assured he would have a high-quality program. He did join the faculty. We negotiated to have research built into the agreement, along with the assurance of quality and the IT systems necessary to track outcomes. These are the important initiatives that come naturally in systems like UC Davis and Lurie Children's, 
but did not here. This created a lucrative program with margins for us, with huge margins for the hospital.

The hospital then recognized that they were losing some other specialists that they had employed. The specialists wanted to work for us. All of a sudden things shifted. They recognized that there was an opportunity to recruit people who attracted the kind of business the system wanted. Now, this isn't full alignment but at least "the light bulb went off," and there is some value in supporting these faculty who are interested in research. It is not getting you to quality, but it is now beyond just brand. Retaining these physicians is important.

There is a lot to learn regarding how incentives are handled at partnering systems. For example, incentives focused on quality are critical. Both Tom and Allison discussed the importance of quality initiatives and how we should be driving this. Community hospitals aren't that driven by quality, unless they understand why it matters. It matters because of market share. Pricing and market share, that is what is important to community hospitals. Market share not only determines their volume; it also determines their market power as well as their ability to set higher prices and protect themselves from being excluded from coverage. Quality matters to rankings, rankings matter to market share. There are other areas where there should be better alignment, cost and value. However, until more dollars are driven through value, this isn't going to happen. This has been one of my real frustrations. I don't think we are aligned with society's interest until we're better aligned on value. Hospitals right now are a drag on value, not the drivers of value. Unless we move towards an insurance product that we manage, value is something where we don't have common ground.

There's also the dance with residents and education with the hospitals.

I can't find a hospital that thinks training medical students is in its' best interest. They recognize it's a cost of doing business for the medical school brand, but the medical students themselves slow down clinical efficiency, add cost, and add risk. They view medical student training as a loss leader to achieve all the other goals.

However, the residents really do need to be seen as valuable to medical centers. Medical centers don't actually understand the value of residencies to clinical care. Medical centers don't understand how valuable residents are in regard to clinical care, not just in terms of outcomes for patients or changing the quality of work, but actually delivering care. Medical centers need to support the training system, but they also need to understand that it's not just a cost center. Somehow, relaying this message, and conducting analyses to highlight the importance of training are useful in creating partnerships.

We have been doing this, and making great progress. All of a sudden, the hospital system wants us to hire more residents than we can possibly manage. We grew from 200 to 315 in the last three years. We are increasing to 400 residents and fellows in the next 3 years. It's lots of growth over a short period of time, because we were able to make the case that training systems help the hospital and its' clinical mission.

Finally, let's review the community piece. This is hard because hospitals already feel like they do a ton for the community. Hospitals provide care that's unreimbursed or under reimbursed, and that's a huge benefit to their community. Academic centers do this more than other medical centers. However, hospitals do receive reimbursement, particularly now in pediatrics. Hospitals do get reimbursed for some of that "unreimbursed" care, through other means.

As we think about expanding those programs, there is often resistance because these initiatives are seen as an additional drag on margin, margin that's used for other important initiatives. So, there are some areas. Alison highlighted a perfect example of one, where working with the community lowers costs and increases margin. There are plenty of other examples. They are just difficult to navigate.

Respite care is another example. For people experiencing homelessness, keeping them in the hospital is really not good for hospitals or for the people who are hospitalized. Hospitals should build respite centers and admit homeless folks there who would otherwise be part of inpatient care because they're receiving IV treatment or other therapies. That's one example. There are many others.

Until we take risks for the population, we are never going to adequately take care of our communities. Without taking risks, care is just going to be as much as is necessary to keep the public thinking that we are aligned and to build the brand, as well as drive more insured folks into the hospital. We are stuck in this reality until healthcare systems actually get paid for value and are truly aligned with the health in a community. Once we achieve that, we will see change. We just have to be real about what makes sense for hospitals and what doesn't.

I agree that COVID did lead to change, at least right now. We are aligned with our hospital in terms of testing, vaccinations, management of acute care, implementing protocols, and conducting research. COVID has been a total game-changer. Sadly, I don't think it's going to stick. I'm just trying to be realistic about this issue. I hope I'm wrong. Some telehealth will stick. I think we will end up going back to our corners after this crisis unless we truly change the underlying incentives.

COVID was an emergency. Everybody comes together in an emergency. We have to change the incentives so that we are better aligned with society's interests, and then once we are, then we'll all be rowing in the same direction. That's my optimistic, but somewhat cynical view of the intersection between these two worlds.

(SD) We are often siloed in our divisions or departments. Could you go into more detail about hospital operations?

(CJ) This is really important to understand. Administrators who run hospitals, people like Tom, are all about results. They're working towards quarterly results, not just financial outcomes, all types of metrics. They track these metrics and are focused on achieving results. In general, we don't do this on the academic side. I'm just putting my academic hat on. The Clinical Research Center is a good example of not being operationally directed. We were not focused on the true costs or the opportunity costs of the space that we were controlling. Research works that way, too. We will just go down a different path because it seems innovative and cool. We have all types of inefficiencies; we are not good managers. That inability to understand our weakness as academic physicians and how it's perceived on the operational side creates a real disconnect and a distrust. We need to understand why an operational focus is so important to our survival and own that on the academic side. I don't know that the hospital administrators will change; we count on them to be that way. I think that a change on the academic side is needed. Honestly, it would be great to have more research and education that's really directed on achieving outcomes.

(SD) Tom, how should children's health care operations be integrated with adult care? It's very different at a freestanding versus a "hospital within a hospital." Where should operations be integrated and where should operations be separate?

(TS) In an integrated system, you are looking for any business that is efficient and this often relates to scalability. The advantage of the pediatric department or children's hospital as part of a broader, integrated system are the efficiencies that are garnered with what we typically call the "back room" support services: information technology, human resources, information management, etc. All these support mechanisms serve more efficiently at scale, and I think in the future we will see independent children's hospitals form regional coalitions around this concept. This is like what we see with single payer systems, Atrium Healthcare, for 
example, or Advocate Aurora, multiple hospitals integrated into a single system to leverage these efficiencies. That's the easy part of why so much of this works to a degree at UCSF, for example.

Leadership, vision, and a willingness on both sides of the street, from the standpoint of the children's center aligning with an adult center, are needed to drive the academic, clinical, and training alignment. You may have joint training opportunities-for example, joint educational sessions. You can think of a million examples, such as cystic fibrosis. What are the clinical areas where you can build a transitional care model? For example, when and how do the pediatricians turn over the cystic fibrosis patient to the adult providers? What about sickle cell disease? What about cancer survival? When you build these relationships within clinical service lines, how do they distinguish themselves in terms of both their outcomes and their specialty within your given market? What are the opportunities to build around the research aspects? What longitudinal data sets can you create, and what insights can you garner in terms of long-standing risks from these opportunities?

We have many of these programs with our adult colleagues at Northwestern Medicine. It takes the leaders to want to really drive towards that vision. I think you can find the common ground usually around a clinical cohort or clinical subspecialty and then leverage the educational opportunities and the research opportunities. What's the investment that may be required to launch that effort? What's the investment that might be necessary in order to partner with adult providers? Might joint investments be needed to fund fellows that can manage that population and transitional care? Can a new revenue stream be created through a transitional care clinic? What kind of funding opportunities might there be for the research that generates that Rol impact? Those would be my suggestions and recommendations.

(SD) Allison, you mentioned a process for estimating the monetary value of research to the healthcare system. All three of you really touched on this issue. Could you elaborate?

(AB) Let's take a comprehensive cancer center. The patient comes to the cancer center because they want to be in a phase 3 trial. The trial brings that patient into the system. Many of the costs are covered by the trial, but not all, particularly if $\mathrm{NIH}$ funded. Then, that patient often will stay in the system. In my own personal experience, the clinical trial patients that I treated stayed in the system and ended up receiving subsequent procedures in the system. Therefore, that's a way that research contributes to a patient acquisition and retention strategy.

Now, you have to understand all the compliance issues and be able to support all of it. This differentiates an academic medical center within a market. Academic medical centers have research that is not at the usual community hospital. This is one of the initiatives, in the middle of COVID, that really differentiated us because we were the only place in town for a long time that had any drugs that could help those hospitalized with COVID.

You may be able to improve your payer mix through these research opportunities. We want patients who come to the comprehensive cancer center and who have choices in whom they choose for medical care. By offering research we really differentiate the academic medical centers as a unique hub of groundbreaking care.

(CJ) On average, for every research grant dollar you receive, you need about 40 to 50 cents extra to pay for that research at an academic medical center. Even with indirects, you don't adequately cover the overhead costs associated with research. In general, it's never in anybody's interest to support research if you just look at the finances. There are a few exceptions.

If you manage multi-center clinical trials, you can make some money. Laboratory-based research loses money. Imaging research sometimes makes money. If you manage those industry trials as an academic research organization, you can make money. There are a few opportunities like these where research, by itself, makes money. As Allison said, it's all about the secondary and indirect benefits of research.

I do think the value of research is substantially under-realized. Cancer is a perfect example. Access to drugs that wouldn't otherwise be available is an advantage. By becoming a cancer center, that's huge in terms of referrals, and brand recognition. Research also impacts ranks and ranks do make a difference. They make a difference in where patients decide to seek care. Ranks make a difference in pricing, too.

I know of two examples where institutions have moved substantially in the US News and World Report Rankings. I'm not encouraging people to focus on rankings. However, these two institutions invested a huge amount in increasing $\mathrm{NIH}$ dollars, aggressively recruiting researchers from other institutions. They were very successful in increasing $\mathrm{NIH}$ dollars and recruitment; it impacted their rankings. I don't have data to prove it, but I'm sure it's impacting their clinical margins as well. I think that illustrates how that connection can benefit hospitals.

(TS) When Clay and I were peers directing the CTSAs at our respective institutions, NCATS [National Center for Advancing Translational Science] conducted this assessment. There is literature regarding the cost/benefit of a research enterprise within an academic medical system. You should pulse check your institution. Is your institution telling you that research is a cost center, as opposed to an investment? When I first arrived at some institutions, I used to hear from the CFO, the pediatric department loses $X$, and the research enterprise loses $Y$. We now say that the institution decides to invest $X$ in the research enterprise and $Y$ in the pediatric service line, because you understand the indirect benefits of these investments. There's some data that suggest that reputational impact is important. As Clay said, it's an imperfect world of rankings, but people that have paid attention to rankings have seen that this investment translates into increased volume demands and regional referral growth.

You can quantify "academic value" from a marketing standpoint. When you have a reputational media hit that lands on the front page of The New York Times, as Allison said, that probably saved $\$ 4$ million in advertising costs for the University of California at Davis. You shouldn't have to go too deep into the weeds of justifying to your medical center the importance of investing in the research enterprise.

I do think the research enterprise can be managed more effectively. I will speak to our enterprise. We had about \$27 million in funding, and we invested $\$ 8$ million in research programs. We now spend about $\$ 6$ million in research programs* and have $\$ 72$ million in research funding (*note, this investment does not include our broader research infrastructure). You can improve that aspect, as Clay said. Clinical trials and clinical studies, if run well, actually can make money for the research enterprise.

Health service research is a worthy investment. As Clay mentioned, there's wet labs for basic science research, and wet labs have to be subsidized from an infrastructure standpoint. Health service investigators, especially those with $\mathrm{NIH}$ funding that have indirect costs, can really do well from a financial investment standpoint. Hospital administrators, who often evaluate these types of investments may see this as a good return on investment. However, when you're thinking about research careers, I want you to chase your passion because we're at such risk in terms of workforce, the pipeline for basic science investigators, and research overall.

(AB) Because we want hospitals to run efficiently, we should insist on the same operational metrics for research as other care the hospital provides. So, we should evaluate metrics such as the time to receiving clinical trial approval, the time you stand up the contract and the time to the first patient enrolled. If you say in the contract, you plan to enroll 100 people, and you enroll two then that is a failed project As leaders, we need to hold people accountable for these issues surrounding clinical trial enrollment. We also need to change the culture. It's critical to think as a 
system, and utilize the EMR and other tools to really drive research. Then you begin to realize the operational efficiencies in the research, enrolling more patients, and moving the science forward.

If you don't have research, you're a community hospital, whether you have a medical school attached to your name or not. Schools of medicine in academic healthcare systems now have an opportunity to make sure that research is part of the discussion with every patient. I think the places that are integrated really have an opportunity to do this. You should insist and demand efficiencies in the research sector, just like in hospital operations.

(SD) Tom, COVID has really affected children differently than adults. What challenges has that brought to Lurie Children's?

(TS) It's brought a lot of challenges to Lurie Children's and other freestanding children's hospitals. Masks work really well for preventing COVID-19, and also work really well for preventing influenza and RSV. In pediatrics, those viruses drive much of our acute care needs in the inpatient setting, respiratory failure in the PICU, and emergency department visits. Infections also lead to otitis media and tonsillitis, and the need for tubes and tonsillectomies. When kids play together and participate in sports, they usually break a lot of bones, that hasn't happened during the pandemic. Orthopedics, ENT, acute care, and emergency department volumes have been impacted for the past several months. I think we're all quoting about the same numbers: down somewhere between $10 \%$ and $20 \%$ for acute care admissions, and down $40 \%$ to $50 \%$ of emergency department admissions. This has had a significant impact on the revenue.

We're fortunate that the federal government has provided some relief. In the integrated programs, pediatrics has described themselves as the anchor that's weighing down the rest of the ship. However, it doesn't matter when you still have a big ship of adult medical and surgical care and adult COVID patients driving overall revenue for that integrated system. We (independent children's hospitals) are only anchors. We don't have a ship dragging us along. We've had to be thoughtful about where we invest our resources at this time. Now, there's a lot of expense you can contract on the volume side. With a variable staffing model, we've contracted FTE. There's also less demand in terms of transaction numbers so we've reduced our fixed staff in those areas. We also have an early retirement program, a more humanistic approach to reducing your total FTE. We didn't replace most of them. This reduced our fixed FTE pool to a point at which we could continue to readily meet operational demand.

Many of our independent peers across the country have the minimal infrastructure, yet there are still expectations to serve our communities and our regions 24/7/365 with complex specialty and critical care. This is part of the argument we're having in terms of additional provider relief to bridge through this time frame until we pivot to alternative care models. We're going to be leaning into digital care broadly, including telemedicine. We also think home patient monitoring or home health care opportunities is the future. We'll also be relying less on inpatient facilities. We are thinking much more about home- and community-based care sites.

Being quite transparent, despite losing $\$ 150$ million from our net operating income last year, we launched the Magoon Institute for Healthy Communities using philanthropic dollars. This is a $\$ 40$ million institute that is our interface with our communities for transforming the care and well-being of children. Let's discuss value-based care. We have a CMS grant that's evaluating an alternative provider payment model for 44,000 kids covered by Medicaid. Transforming the healthcare system to value-based is an important investment.

We have also recruited a top pediatric cardiothoracic surgeon, not only because his clinical outcomes were strong, but also because of his research; he is conducting a phase 2 stem cell therapy trial for myocardial support. Investing in this type of innovative research program, despite pressing financial times, was important because we know this will pay significant dividends in the future.

I did want to discuss the actual cost estimate of a research enterprise. It doesn't happen often and you have to be strategic, but leveraging intellectual property from the research enterprise for licensing can serve as a revenue stream. You may decide to do this and invest some to build tech transfer capabilities, or align with your academic partners. This is why we may be investing in intellectual property development as a research enterprise.

(SD) Clay, running clinical operations frequently trumps academic achievement and selection processes for division leadership. How do we ensure that the research mission not only survives, but thrives with a diminishing fraction of physicianscientists promoted to leadership roles?

(CJ) I think this is probably location and specialty-specific because my observation at UCSF was the opposite. Division leaders were often researchers that really didn't understand how to be managers or oversee clinical operations. If you evaluate revenue streams in these positions, there's zero for education, a small amount for research, and a lot more for clinical. As part of these leadership positions, the person really has to understand clinical operations. I would encourage this. This goes against that notion of "focus, focus, focus" as a researcher. It's an important question. We don't want to lose the essential commitment to research. We must balance clinical care, research, and education. We don't think about this on the community side. Tom's story is heart-wrenching. On the other hand, Tom, I'm sure it makes your stomach turn a little to say, that you're disappointed that influenza cases are down. I mean, it's great for kids. When your business is down, it's good for kids. I also think we need innovators, and they can come from a research background or other backgrounds. We need innovators in leadership positions who are willing to take risks.

Tom's description of moving forward with a new plan and value-based healthcare, that's exactly the kind of innovations that we need. We want to be aligned with the interests of the children in our communities. The finances, the way they're managed, are against this alignment. The pace of innovation on the community side and on the clinical side really impacts care delivery; it's too slow and not aligned with the community's best interests. As soon as finances align with quality and outcomes, including prevention, care delivery will improve. We do need innovators in these roles and researchers are often great innovators. We need people to push that agenda forward.

(SD) Each of your schools have a Dean's tax on clinical revenue. How large is it, and how is it used?

(AB) We're in the middle of a transition to funds flow. People need to understand how the margin of the clinical mission, support the academic piece, and the amount of funds that flow. I would not compare Dean's taxes because if you've seen one financial model at an academic medical center, you've seen one financial model. Everybody has a different opinion about funds flow models. The whole benefit of the concept is that it raises all boats. So, the person who cares for the geriatric patient and only does $E$ \& $M$ work is paid for keeping these patients healthy and out of the hospital. The proceduralist benefits from the person who is doing only $E$ \& $M$ work. This requires culture change, and it has to "right size" different areas. I do think that is the value of being in an integrated model. This work at Wake Forest took many years. As a neurology chair, when I arrived, people were grossly underpaid for their $E \& M$ work. The funds flow model fixed this problem and teams were more patient-centered around the disease state. It requires a lot of change management, transparency, conversation, and culture change.

You mentioned intellectual property earlier. You invent something, and before you publish it in Nature, you speak to an attorney, who's going to ask lots of questions. Why in the world 
would you do that when Nature's waiting? You do this because it's a discovery that can be leveraged to help pay for and build infrastructure. I think all of this truly requires culture change.

(CJ) Every place is so different so it's really hard to answer this question. There's not really a single calculation at most places. For us, it depends on the program. Some have high margins associated with them and some have negative margins. We cross-subsidize those with negative margins. We don't pull all this together and say that is the tax. We don't push it down to the departmental level.

It would be awesome to have an institution have clinical revenues cross pay for every other mission, but this is often not possible. However, clinical revenues often do pay for some of the other missions. You rely on the clinical revenue and you subsidize the research and education missions based on mainly the clinical revenue. Institutions balance missions this way.

(TS) This may be provocative, but we don't have a Dean's tax. We return $5 \%$ of the professional fee revenue to our clinical departments. Is that enough? The hospital invests substantially more in the "academic missions". My approach is to ask what investments do you need to achieve the goals I'm asking you to accomplish?

I will also add to the question about choosing divisional leadership based on the skillset of being strong clinical managers instead of physician scientists. I disagree that there has been a switch away from physician-scientists. It's much more important for me to identify a leader who embraces and understands the importance of each of the missions: clinical, research, education, and advocacy. I want a leader who knows how to really lead and advance these missions.

I, as a hospital administrator, can provide them the management support to take care of the clinical operations. I can't provide them a research or teaching expert. We build our leadership recruitments around those that can lead our academic enterprises.

(SD) There's evidence that academic health centers are more successful with a physician CEO in place, as opposed to a nonphysician CEO. What do each of you think?

(TS) My ego recuses me from answering that question directly. I don't have a good answer, since I've been in the CEO role for only 15 months. I never aspired to this role, and I'm learning as I serve. I would say the advantages are that I grew up as an academic pediatric investigator and understand the importance of healthcare system alignment with the academic enterprise. I may be better at judging the efficiencies of the operations of some of the academic enterprise. There are also some disadvantages in terms of not understanding the finances, contracting models, partnership health systems. That's why you build a team. No one person is responsible for leading an organization, a division or department. You build the expertise within the team. Does it need to be a physician running an organization? No. Do you need physician input? You sure do! You have access to this expertise, so you make sure you invite physician input, and respect it.

(AB) I don't think you can come down on either side of this question. We need leadership that appreciates all we've discussed today. Leaders need to understand research, education, clinical care, and quality. If we ever really implement value-based payments, this approach is at the heart of all that we do.

It's incumbent on physicians to learn about the business of medicine. That doesn't mean you have to attend graduate school, and you don't need to know how to complete fancy spreadsheets. You do need to understand operations, throughput, and why it's important to decrease the length of stay. Smart business practices don't just belong in hospitals. Smart business is part of the academic medical center, education mission, research mission, and hospital. Also, always hire people smarter than yourself.

(SD) Systems that support academic missions often have a "survival of the fittest" mentality. This may lead to difficulties maintaining collaborations with other academic programs. How do you keep these competing groups aligned and minimize the animosity that can be generated in this type of environment? This can occur in cities where there's many different medical centers.

(CJ) When I was in California, this was an issue. I was working on multi-institutional alignment as the head of the CTSA. I was trying to encourage institutions, who were accustomed to competing, to work together, including Stanford, UCLA, UCSF, and USC. Institutions go to battle. Individuals don't really care. CEOs, branding and communications people, and other administrators may not like each other. When you're working for a common purpose, such as increasing our knowledge about an illness or dealing with a public health issue, this really draws people together. This is one of the wonderful things about being in academics: we can coalesce around purpose. That's the way you drive alignment. Don't get permission from the CEOs if they're fighting; just do the work. It's fun and you know these are your colleagues. You all want to arrive at a solution. We had success in California using this approach.

(TS) I am worried about all these challenges. There is the challenge of many centers within cities, compression within those markets, and the challenges of increasing sub-specialization of pediatric care. This will lead to care being located in a handful of centers. When you evaluate the impact of case volume to trainee exposure, we have to question if students are really receiving enough clinical exposure during pediatric clerkships. This is relevant to residency and fellowship programs too. I don't know exactly where the training experience is going to occur in the future for smaller programs. While I think there are important opportunities for partnership and collaborations in regard to research and quality data sharing, there's going to be an increasing challenge when creating partnerships for education and training.

This accelerates the challenge of our workforce. APS and SPR are committed to tackling the issues associated with our subspecialty workforce challenges. I look forward to working with these two societies as a CEO. I want to work with these two wonderful societies to address these pediatric workforce challenges.

\section{COMPETING INTERESTS}

The authors declare no competing interests.

\section{ADDITIONAL INFORMATION}

Correspondence and requests for materials should be addressed to Thomas Shanley.

Reprints and permission information is available at http://www.nature.com/ reprints 